\title{
Impact of temperature and precipitation extremes on the flowering dates of four German wildlife shrub species
}

\author{
Jonatan F. Siegmund ${ }^{1,2}$, Marc Wiedermann ${ }^{1,3}$, Jonathan F. Donges ${ }^{4,5}$, and Reik V. Donner ${ }^{1}$ \\ ${ }^{1}$ Research Domain IV, Transdisciplinary Concepts \& Methods, Potsdam Institute for Climate Impact Research, \\ Telegrafenberg A31, 14473 Potsdam, Germany \\ ${ }^{2}$ Institute of Earth and Environmental Science, University of Potsdam, Karl-Liebknecht-Str. 24-25, \\ 14476 Potsdam-Golm, Germany \\ ${ }^{3}$ Department of Physics, Humboldt University of Berlin, Newtonstraße 15, 12489 Berlin, Germany \\ ${ }^{4}$ Research Domain I, Earth System Analysis, Potsdam Institute for Climate Impact Research, Telegrafenberg A31, \\ 14473 Potsdam, Germany \\ ${ }^{5}$ Stockholm Resilience Centre, Stockholm University, Kräftriket 3B, 11419 Stockholm, Sweden
}

Correspondence to: J. F. Siegmund (jonatan.siegmund@pik-potsdam.de)

Received: 13 September 2015 - Published in Biogeosciences Discuss.: 16 November 2015

Revised: 25 August 2016 - Accepted: 20 September 2016 - Published: 6 October 2016

\begin{abstract}
Ongoing climate change is known to cause an increase in the frequency and amplitude of local temperature and precipitation extremes in many regions of the Earth. While gradual changes in the climatological conditions have already been shown to strongly influence plant flowering dates, the question arises if and how extremes specifically impact the timing of this important phenological phase. Studying this question calls for the application of statistical methods that are tailored to the specific properties of event time series. Here, we employ event coincidence analysis, a novel statistical tool that allows assessing whether or not two types of events exhibit similar sequences of occurrences in order to systematically quantify simultaneities between meteorological extremes and the timing of the flowering of four shrub species across Germany. Our study confirms previous findings of experimental studies by highlighting the impact of early spring temperatures on the flowering of the investigated plants. However, previous studies solely based on correlation analysis do not allow deriving explicit estimates of the strength of such interdependencies without further assumptions, a gap that is closed by our analysis. In addition to direct impacts of extremely warm and cold spring temperatures, our analysis reveals statistically significant indications of an influence of temperature extremes in the autumn preceding the flowering.
\end{abstract}

\section{Introduction}

In comparison to geological timescales, ongoing climate change is extraordinarily fast (IPCC, 2013). The associated changes in meteorological conditions, which are among the main driving factors for plant growth, are a huge challenge for terrestrial ecosystems: in some cases, the quick changes may exceed their ability to adapt to the new conditions, leading to severe temporal or spatial mismatches between interacting/symbiotic species that may cause critical disruptions of the food chain and thus affect population size and health of both species.

Beyond the gradual change in the mean climatology of Europe, the spatial extent, intensity and frequency of extreme climate events like droughts or heatwaves have also markedly increased over the past decades (Coumou and Rahmstorf, 2012; Tank and Konnen, 2003; Luterbacher et al., 2004; IPCC, 2013). Both the probability of occurrence and the amplitude of many types of climatic extremes have been rising (Fischer et al., 2007; Barriopedro et al., 2011; Petoukhov et al., 2013; Seneviratne et al., 2012) and are projected to increase further (Stott et al., 2004; Rahmstorf and Coumou, 2011; Petoukhov et al., 2013). Especially during recent years, extreme summer temperatures have been observed which were clearly beyond the limits of previously observed extreme values. Specifically, examples like the European heat- 
wave in 2003 (Schaer et al., 2004; Luterbacher et al., 2004; Garcia-Herrera et al., 2010) or the Russian heatwave in 2010 (Trenberth and Fasullo, 2012) by far exceeded historical extreme values of the past 500 years.

In terms of water availability, past and ongoing trends of heavy rainfall events strongly depend on region and season (Tank and Konnen, 2003; Lupikasza et al., 2011; Coumou and Rahmstorf, 2012; Haylock and Goodess, 2004), whereas future projections suggest increases in those events' frequency and intensity for most parts of Europe (Kundzewicz et al., 2006; Kysely et al., 2011; Rajczak et al., 2013). In turn, droughts as a combination of high temperatures, low precipitation and high evapotranspiration only show low to moderate positive trends for central Europe during the last 60 years (Spinoni et al., 2015b; Gudmundsson and Seneviratne, 2015). For the future development of drought intensity and frequency over central Europe, trend estimates have provided ambiguous results (Spinoni et al., 2015a).

The effects of climate extremes on terrestrial ecosystems are diverse and highly complex and may lead to unprecedented outcomes (Frank et al., 2015; Reichstein et al., 2013). Besides direct impacts, there is a growing body of evidence that climate extremes can critically disturb sensitive ecological equilibria (Parmesan, 2006) and mutualisms (Rafferty et al., 2015). The effects of temporal displacement or even absolute failure of flowering and fruit ripening of food plants for nectarivores, small mammals and birds are important examples (Law et al., 2000; Jacobs et al., 2009). A rapid population decline up to species extinction due to phenological mismatches between plant and pollinator has already been demonstrated (McKinney et al., 2012; Burkle et al., 2013; Kudo and Ida, 2013). The resulting damage on the affected population could propagate through the ecosystem and endanger its structure, dynamics and stability (Post and Stenseth, 1999; Parmesan et al., 2000; Parmesan, 2006; Augspurger, 2009).

A widely used source of long-term observations allowing us to study the inter-annual variability of plant growth dynamics is the timing of phenological phases. From several studies, it is known that the phenological phases of most central European plant species experience systematic, gradual changes related to climate change. Especially the change in temperature plays an important role for long-term variations in the dates of foliation, flowering and leaf colouring (Ahas et al., 2000; Sparks et al., 2000; Sparks and Menzel, 2002; Menzel, 2003; Cleland et al., 2007; Schleip et al., 2012).

However, it is likely that seasonal temperature extremes can affect terrestrial ecosystems much more strongly and more directly than gradual changes (Easterling et al., 2000; Jentsch et al., 2007, 2009; Zimmermann et al., 2009; Menzel et al., 2011; Nagy et al., 2013; Reyer et al., 2013). Associated with extreme weather conditions, flowering dates of temperate species have been observed to be shifted by up to 1 month or to have even failed completely (Nagy et al., 2013).
Unlike for temperature extremes, the possible impact of drought or heavy precipitation events on plant flowering is less well understood. So far, only few studies have explicitly addressed this question, and those that have are of an experimental nature only. The experiments of Nagy et al. (2013) and Jentsch et al. (2009) found significantly delayed flowering dates of Genistra tinctoria after drought treatment. On the other hand, Nagy et al. (2013) also found that the average flowering date of Calluna vulgaris was not significantly affected by drought. In a similar study, Prieto et al. (2008) observed no shift in the flowering dates of Erica multiflora related to drought. Heavy rainfall did not effect flowering time at all in the experiments of both Nagy et al. (2013) and Jentsch et al. (2009).

In general, the reaction of flowering to climate extremes has so far mainly been analysed for individual events (Luterbacher et al., 2007; Rutishauser et al., 2008) or with experimental setups (Prieto et al., 2008; Jentsch et al., 2009; Nagy et al., 2013). Systematic studies exploiting existing large-scale spatially distributed data of phenological phases by means of sophisticated data analysis methods are scarce. As one notable exception, Menzel et al. (2011) presented an in-depth analysis of the influence of warm and cold spells on crop plant phenology over Europe. However, since agricultural crops are often subject to specific treatments (which have changed over the past decades), these results are not directly transferable to wildlife plants, for which a corresponding study is still missing.

In order to close this research gap, we investigate the individual influence of extremely high- and low-temperature and precipitation events on the flowering dates of four central European wildlife shrub species, using a phenological data set covering the period from 1950 to 2010. In contrast to other recent studies (e.g. Rybski et al., 2011), we intentionally focus on flowering as a single phenological phase with paramount ecological importance. Moreover, we select just four shrub species (see Sect. 2) as a case study to address the following research questions:

- Do the flowering dates of these shrub species systematically react to temperature and/or precipitation extremes?

- Which species are more/less susceptible?

- Do these effects differ by region?

The remainder of this paper is organized as follows: after a description of the phenological and meteorological data sets under investigation, the approaches of extreme-value definition as well as the methodology of event coincidence analysis are described in Sects. 2 and 3, respectively. Subsequently, the results of our study are presented in Sect. 4 and further discussed in Sect. 5. We conclude this paper with a short summary of the results in Sect. 6 . 


\section{Data}

\subsection{Meteorological data}

As a climatological reference data set, we use an ensemble of homogenized and expanded daily mean temperature and precipitation time series from Österle et al. (2006), which are based on meteorological stations operated by the German Weather Service (DWD; Deutscher Wetterdienst, Offenbach, 2009). While the precipitation data are directly based on observations made at all considered stations, mean temperatures partially involve a sophisticated spatial interpolation from a set of fewer stations with direct measurements (Österle et al., 2006). Both data sets are commonly employed as a benchmark data set for assessing the performance of hindcast simulations of regional climate models (German baseline scenario). The data set covers the time interval from 1950 to 2010 and comprises 1440 stations distributed across Germany as well as a set of stations located in the adjacent regions of some of its neighbouring countries.

\subsection{Phenological data}

As a source of information on the reactions of terrestrial ecosystems to climatic drivers, we use the German Plant Phenology Data Set, provided by DWD (http://www.dwd.de/ phaenologie). This data set contains the Julian days of the occurrence of several phenological phases. Besides 22 fruit species and 22 crop types, the data cover 37 wildlife species at 6525 stations distributed over all of Germany for a period from 1951 to 2013. However, the actually available time series length strongly varies by station. While some stations have series covering the full considered time span, others contain just a few or even only one observation per plant species and phenological phase. Due to these different time series lengths, we select only those stations for our further analyses which contain at least 40 years of observation between 1951 and 2010.

In this work, we analyse the flowering dates of four of the most abundant German wildlife shrub species: lilac (Syringa vulgaris L.), elder (Sambucus nigra L.), hawthorn (Crataegus monogyna (Jacq.)/Crataegus laevigata (Poir.) DC) and blackthorn (Prunus spinosa L.). These four shrubs are representative of the regional vegetation. Moreover, they are characterized by a usually large amount of flowers during early to late spring and constitute important components of their local ecosystems, which are in some regions key for local insect, bird or small-mammal populations. For example, hawthorn and blackthorn are visited by 149 and 109 insect species, respectively, with around 100 lepidoptera species among them (Southwood, 1961). In contrast, elder is of lower importance for insect species (only around 20 species are known to depend on elderflowers or fruits; see Duffey et al., 1974), but it is an important food source for numerous birds during summer and autumn due to its high amount of very nutritious

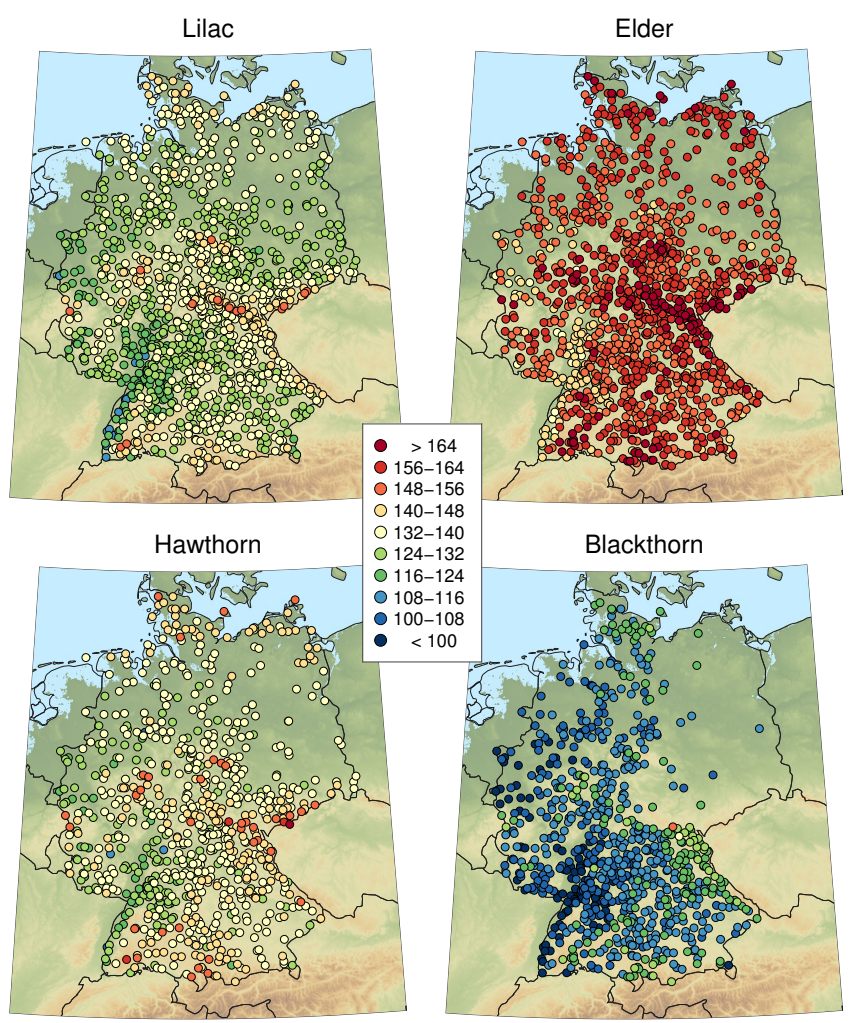

Figure 1. Mean flowering dates (Julian days) of the four analysed shrub species. The figure only shows those records that contain at least 40 observations.

berries (Atkinson and Atkinson, 2002). Other shrub species contained in the DWD data set do either not fall into the same category regarding massive flowering and spatial distribution or the available amount of data is considerably smaller. Therefore, these additional data are not used in the present study, which focuses on the aforementioned four species as illustrative examples with reliable data.

The mean flowering times of the considered shrub species range from early April (blackthorn) to May (hawthorn and lilac) to mid-June (elder); see Fig. 1. The distributions of the flowering dates of all four species are, however, very wide. Flowering can even occur 1-2 months earlier than normal under certain conditions, which shall be further explored in the course of this work. Due to the selection criterion of at least 40 years of data (at most 20 missing years of observations), the data set is strongly reduced to about 1000 recording sites per plant, and the spatial distribution of the corresponding phenological stations becomes much more heterogeneous, with larger gaps existing especially for blackthorn in northeastern Germany (Fig. 1). 


\section{Methodology}

\subsection{General relationship between flowering dates and meteorological conditions}

Before explicitly focusing on the timing of extremes, it is reasonable to address the general dependency between spring temperature/precipitation and the flowering dates of the four shrub species, taking the full empirical distribution of the different observables into account.

For this purpose, the raw data described in the previous section are analysed according to the following scheme:

- The flowering dates of each individual station are normalized according to their respective mean and variance, using a classical $z$ score approach. This normalization is necessary for the following investigations, since the individual study sites differ strongly in their year-to-year flowering date variability, so that absolute changes cannot be compared between two stations. For example, a shift of flowering by 10 days might be important for site $\mathrm{A}$, while having only a minor impact (i.e. it can still be within the "normal" variability range) for station B. Hence, our normalization procedure allows for inter-comparability between the results of different phenological stations at the cost of losing explicit date information, which is otherwise practically important for common ecological studies. A combination of both viewpoints might be helpful to get as much information as possible out of the given data set. However, given the focus of the present study, we restrict ourselves to the consideration of normalized flowering dates in the following.

- For each meteorological station, the temperature and precipitation observations are consolidated to mean daily spring temperature and the sum of spring precipitation (using daily data for the Julian days 31 to 120 of each year), resulting in time series with one value per year for each station. The resulting time series are transformed into $z$ scores following the same approach as for the flowering dates.

- The $z$ scores of temperature and precipitation from all considered stations are categorized into 20 equiprobable groups according to the 20 inter-percentile classes of $5 \%$ width each.

- The distribution of the flowering dates of each shrub species taken from all stations is evaluated separately for the 20 different categories according to the respective assignment of the associated meteorological observations.

\subsection{Definition of extreme values}

\subsubsection{Phenology}

In order to take a sufficiently large set of events into account that allows us to draw statistically justified conclusions, we define a flowering date earlier than the empirical 10th percentile of all recorded values at a given phenological station to be extreme. Hence, each time series of flowering dates has an individual absolute threshold date for the definition of an early flowering event. This approach is chosen since the timings of the phenological phases of every station can crucially depend on local conditions like altitude, exposition, water availability, etc. Since the time series lengths differ between the different phenological records (40 to 61 observations), this approach also leads to different numbers of extremes in each time series. The definition of extreme late flowering dates is performed in full analogy using the 90th percentile.

\subsubsection{Temperature and precipitation}

In order to obtain information on temperature and precipitation extremes that is directly comparable with the phenological information, a three-step treatment of the available continuous daily meteorological records is necessary, which is detailed below:

Spatial interpolation As a first step, for each phenological station used in this study, we create one daily mean temperature/precipitation series by spatial interpolation of the existing observational records. For this purpose, we apply inverse geographical distance-weighted mean interpolation, using the four closest meteorological stations surrounding each site with phenological recordings. Since we are only interested in the timing of (local and seasonal) temperature (precipitation) extremes rather than the associated explicit values of the respective variables, we do not explicitly take other covariates into account, although being aware of their actual relevance for the specific timing of flowering. Due to the different spatial coverage of phenological data for the four considered plant species, this approach results in four new temperature (precipitation) data sets to be further exploited as described in the following.

Temporal averaging Extreme climatic conditions present for just a single day may not be sufficient to trigger a detectable ecological response like an anomalous date of flowering (Menzel et al., 2011). In turn, given the common timescales of plant physiological processes, it appears reasonable to consider extremes in the mean climate conditions taken over a certain period of time. The aspect of the crucial temporal duration of a climatic extreme event to influence flowering time is of special interest for the interpretation of the impact of climate change scenarios on plant flowering. Accordingly, in 
a second step of preprocessing, we calculate the average daily mean temperature (daily precipitation) for running windows in time. In order to explicitly study the effect of the averaging timescale and potentially demonstrate the robustness of the obtained results against the specific choice of windows, we consider three different window sizes of 15, 30 and 60 days. These windows are moved along the time series with a step size of 1 day. For the 15- and 30-day periods, these windows start on 1 January of the year prior to the flowering and extend up to 1 December of the subsequent year (700 steps). For the 60-day window, the last step starts on 1 November $(670$ steps). This procedure leads to "window-mean temperatures/precipitation", resulting in 700 (670) values for each year from 1951 to 2010 and for each phenological station. Notably, we use an unweighted averaging, giving the same weight to all observations within a given time window.

\section{Definition of temperature/precipitation extremes}

Before defining extreme window-mean temperatures/precipitation, we account for the numerous missing data values of the phenological data set by discarding the meteorological information for all those years, where the corresponding phenological information is missing. We then identify those among the remaining windows for which the corresponding value exceeds the 90th percentile (or falls below the 10th percentile) of all windows of the same size and time period at one station and consider them as extremes. By using this approach, the seasonal variability of temperature and precipitation is already included in the threshold definition, so that no further preprocessing (e.g. calculation of climatological anomalies or $z$ scores) is necessary.

In the case of precipitation, our approach is equivalent to the calculation of the standardized precipitation index (SPI), resulting in 15-day SPI, 30-day SPI and 60-day SPI values. The application of the 10th and 90th percentile then produces (extreme) events corresponding to the SPI category "moderately dry/wet" (WMO, 2012).

\subsection{Event coincidence analysis}

\subsubsection{Basic idea}

To detect and quantify a possible statistical interrelationship between extreme seasonal temperatures (or extreme precipitation) and extreme flowering dates, we apply event coincidence analysis (Donges et al., 2011, 2016; Rammig et al., 2015), a novel statistical framework which allows the identification of non-random simultaneous occurrences of events in two series. For this purpose, for each considered phenological station we convert the two time series (window-mean temperature/precipitation and flowering date of the given

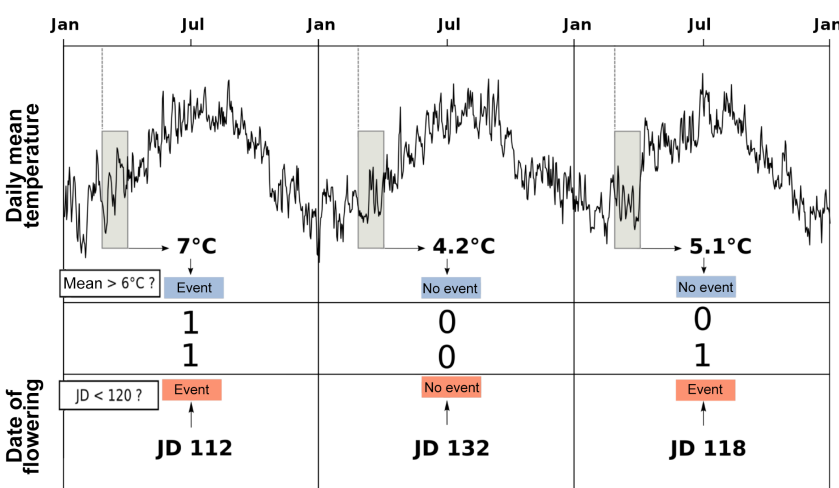

Figure 2. Schematic illustration of the event coincidence analysis used in this work. Upper and lower panels depict the approaches used for defining events based on climatological (daily mean temperature or precipitation) and phenological information (Julian day of flowering), respectively. For the climate data, windows covering the same time interval during each year are fixed for computing window-mean values. The width and location of these windows are varied throughout the analysis as described in the text. Extreme conditions are defined by the exceedance of certain quantiles of the respective variable of interest (flowering time or window-mean value of the considered meteorological variable for the specified window width and position, i.e. one value per year)

year) into binary vectors, representing time steps with or without such extreme conditions, as explained above (see Fig. 2 for a schematic illustration of the approach). Subsequently, we count the number $K_{\text {obs }}$ of simultaneous events (in the following referred to as "coincidences"). In order to assess the significance of the associated normalized coincidence rate $\kappa_{\mathrm{obs}}$, we compare $K_{\mathrm{obs}}$ with the probability distribution of the number of coincidences that would result from two independent Poisson processes with the same event rate as the series under study (see the Supplement accompanying this paper for further information). Further details on this significance test, its limitations and possible alternatives can be found in Donges et al. (2016); Siegmund et al. (2016b).

By performing event coincidence analysis between flowering time and window-mean temperature/precipitation for different time windows before the typical flowering date, we can take possible lagged responses of the plants into account. In turn, studying coincidences between extremes of, e.g., flowering dates and future temperatures (which cannot causally be linked to the flowering), provides a simple test of the reliability and robustness of the obtained results (see Figs. 4 and 5).

\subsubsection{Differences with respect to correlation analysis}

It is important to highlight that there are several fundamental differences between event coincidence analysis and classical correlation analysis as employed in most recent studies on the impact of climate change on terrestrial ecosystems. While we only provide a brief discussion here, more details 
can be found in the Supplement. In the latter, we also provide some numerical examples using artificial data sets as well as selected records studied in this work to support the complementary nature and added value of the methodology used in this work.

The most notable differences between event coincidence analysis and correlation analysis are as follows:

Conceptual viewpoint There are two fundamental differences between correlation analysis and event coincidence analysis already at the conceptual level. Correlation analysis generally takes all observations of two data sets (with the exception of preprocessing for removing possible outliers) with the explicit values of the two variables under study (after possible normalization) into account to provide an estimate of the strength of the statistical interrelationship. In turn, event coincidence analysis considers a distinctively different aspect of a possible statistical interrelationship by making use of preselected data points only (in our case, values in the uppermost/lowermost percentiles of the distributions of the variables under study) and does not use explicit values of the respective observables, but only information about the timing of these values. In this regard, event coincidence analysis reduces the effective sample size by considering only subsets of observations with distinct features, which may help to focus on a specific research problem where only this subset is of particular interest.

Linearity/monotonicity assumption The basic idea beyond correlation analysis in the classical (Pearson) sense is estimating the strength of a statistical interrelationship between two variables by considering the goodness of fit of a linear regression model linking both variables, commonly relying on a Gaussianity assumption that can only be relaxed in the case of sufficiently large sample sizes. The linearity assumption can be relaxed to a monotonicity condition when replacing the explicit time series values by rank numbers, leading to the well-known Spearman rank-order correlation. However, fully non-linear statistical interrelationships (like simple quadratic dependencies) cannot be properly captured by correlation analysis, but require the utilization of more general concepts of statistical interrelationship like mutual information, the estimation of which, however, requires much larger sample sizes. In turn, event coincidence analysis does not make any assumption about the actual functional shape of the interrelationship between two variables beyond two specifically defined "classes" of observations coinciding in terms of their timing. This is commonly the case if a strong linear relationship is present; however, due to the reduction of the effective sample size, one may also find statistically insignificant event coincidence rates in the case of relatively large correlation coefficients or, in turn, observe high coincidence rates when correlation coefficients indicate negligible statistical dependence. We provide numerical examples for both cases in the Supplement accompanying this paper.

Statistical significance Finally, the notion of statistical significance used by both methods is distinctively different due to the very different types of null hypothesis and test statistics derived from the statistical quantity of interest. For correlation analysis, a $t$ test (or Mann-Kendall test in the case of rank-order correlations) is the most common tool of choice, whereas these tests are not applicable in the event coincidence analysis framework. Instead, the test statistic of the latter method is based on a binomial distribution (see the Supplement and Donges et al., 2016, and Siegmund et al., 2016b, for details), the critical values of which are either analytically computed or numerically estimated by means of bootstrapping approaches. In the end, since event coincidence analysis focuses on small subsets of the data under study and thus operates on a much smaller sample size than correlation analysis, its significance statements are more restrictive but also more uncertain (i.e. we have both a lower specificity and lower sensitivity of the associated significance test).

In summary, these three major differences raise the expectation that information on significant event coincidence rates cannot be directly inferred from the presence of significant linear correlations. Following this, event coincidence analysis may actually provide additional information on the emergence of extraordinary reactions of wildlife plant flowering on meteorological stressors that could be discarded by correlation analysis. In the Supplement, we provide some examples highlighting the differences between the results obtained using both methods for the given data sets.

\subsection{Multiple testing}

Our sliding window approach using mutually overlapping time intervals with evident serial correlations of the meteorological variables of interest leads to a multiple testing problem. The standard approach for dealing with such problems would be a Bonferroni adjustment of the significance level (Shaffer, 1995). Although being aware of this approach, in this study such an adjustment is not considered since the analysis modified in this way would not provide practically useful results in the context of our research agenda. Specifically, our decision against a Bonferroni adjustment to be used in this study follows the arguments raised by Perneger (1998):

- The Bonferroni adjustment is based on one general null hypothesis, i.e. that all individual null hypotheses are true simultaneously. In our present study, it is not intended to state that all shrub stands of one species are 
prone to climate impacts in the same manner, which cannot be expected realistically. In turn, our analysis rather seeks to identify general tendencies, which may have multiple individual exceptions. In a similar spirit, our sliding window approach with respect to the meteorological conditions is used as a purely exploratory tool for identifying time windows during which extraordinary meteorological conditions have the strongest relevance for the timing of subsequent plant flowering. In turn, we do not intend to primarily interpret the performed statistical tests in a confirmatory sense.

- Using a Bonferroni adjusted significance level implies that the interpretation of a finding depends on the number of other tests performed. Since the number of phenological stations and, hence, the number of significance tests in this study is larger than 1000 for almost all shrub species, the Bonferroni adjusted significance level would be very close to 0 . Thus, such an adjustment cannot be of practical interest for the interpretation of the results of our analysis, since all interdependencies would be discarded by a test with the accordingly corrected significance levels. Or, put differently, "The likelihood of type II errors is also increased, so that truly important differences are deemed non-significant" (Perneger, 1998).

\section{Results}

\subsection{General relationship between flowering dates and meteorological conditions}

Figure 3 illustrates the distribution of standardized flowering dates ( $z$ scores) of all four shrub species for the twenty $5 \%$ intervals of the two considered meteorological variables. Here, the time span taken for the definition of a "mean spring temperature" and "spring precipitation sum" is related to the typical flowering dates of each species: Julian days (JDs) 59119 for lilac and hawthorn, JDs 89-149 for elder, and JDs 39-99 for blackthorn. As expected, our results demonstrate a generally very strong negative temperature effect on flowering (i.e. higher spring temperatures foster earlier flowering). A more detailed inspection also reveals that the dependency between spring temperature and flowering is monotonic but slightly non-linear. Specifically, the slope of the estimated statistical relationship increases markedly for spring temperatures above the 90th percentile for all four species. Besides this, the delaying effect of particularly cold spring temperatures on flowering times is slightly stronger than the average dependency (slope) for lilac and hawthorn. In contrast to the findings for temperature impacts, precipitation has hardly any systematically positive or negative influence on the flowering dates, only elder flowering dates seem to be delayed in years with a high spring precipitation amount.
From the above results, we expect that extremely wet or dry conditions during spring may not have a marked influence on the timing of flowering of the four considered shrub species, while extraordinarily high or low spring temperatures could have a stronger effect on the flowering dates than could simply be expected from the outcomes of correlation analysis. In the following, we will examine the validity of these expectations in more detail by means of event coincidence analysis.

\subsection{Coincidences with positive temperature extremes}

We start our detailed investigations on the impact of extraordinary warm spring temperatures by considering lilac as an example for illustrating the performance of our method in practice. Figure 4 demonstrates the existence of significant coincidence rates between very early lilac flowering and extremely warm window-mean temperatures for three different window sizes and all windows from 1 January of the preceding year to 1 December of the year of flowering. Significant coincidences at a confidence level of $\alpha=0.05$ are displayed in red, those that are also significant at $\alpha=0.01$ in black.

For all three window sizes, a maximum number of stations with significant coincidence rates is found during the spring months, especially around March and April. For time windows after the typical flowering time in May, there are generally much fewer indications of corresponding interrelationships than for windows before May. Note that due to the statistical nature of the employed analysis methodology, there are always individual stations exhibiting a significant number of coincidences just by chance, even if there cannot be a causal link between the considered events. This is due to the very small number of events the analysis for each individual station is based upon, i.e. the significance of the results for a single given station is practically irrelevant and becomes only statistically meaningful if the whole ensemble of stations (or a sufficiently large subset thereof) is considered. For example, at a $5 \%$ confidence level, we may expect that at most $5 \%$ of the stations show false positive results (i.e. individually significant results that occur simply due to chance only; same at $1 \%$ level), which is about the order of the maximum numbers of stations with significantly many coincidences observed after May. Hence, this behaviour is to be expected.

Regarding the spatial distribution of stations with significant coincidence rates, we do not observe any systematic latitudinal trend, with one exception: at the northernmost stations, the timing of significant coincidence rates between early flowering and extreme positive temperature anomalies tends to extend further into the late winter than for the more southern stations.

Considering time windows from the previous year, we find some indications of summer (60-day windows) and autumn (15- and 60-day windows) temperature extremes to significantly coincide with early flowering in more cases than is to 
Temperature
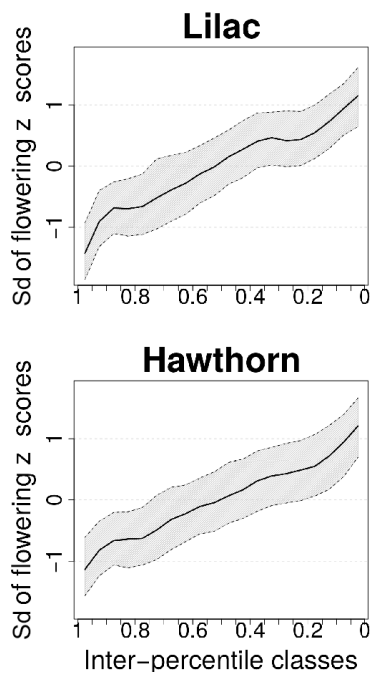
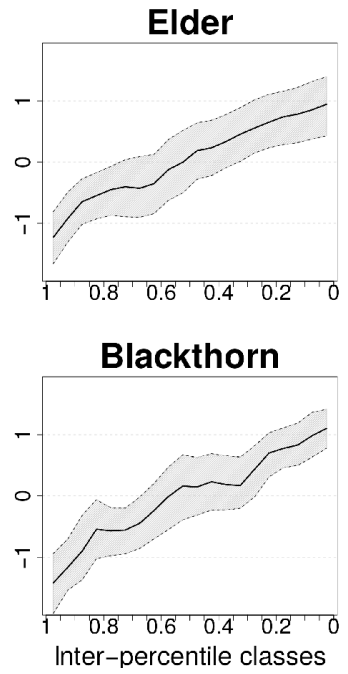

Precipitation
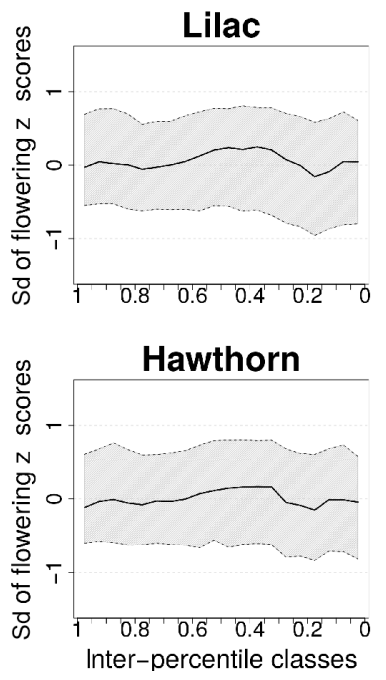

Figure 3. Distribution of standardized flowering dates (median plus 25\%/75\% interquartile range) of the four shrub species in dependence on spring mean temperature and spring precipitation sum. Note the inverted direction of the $x$ axes.
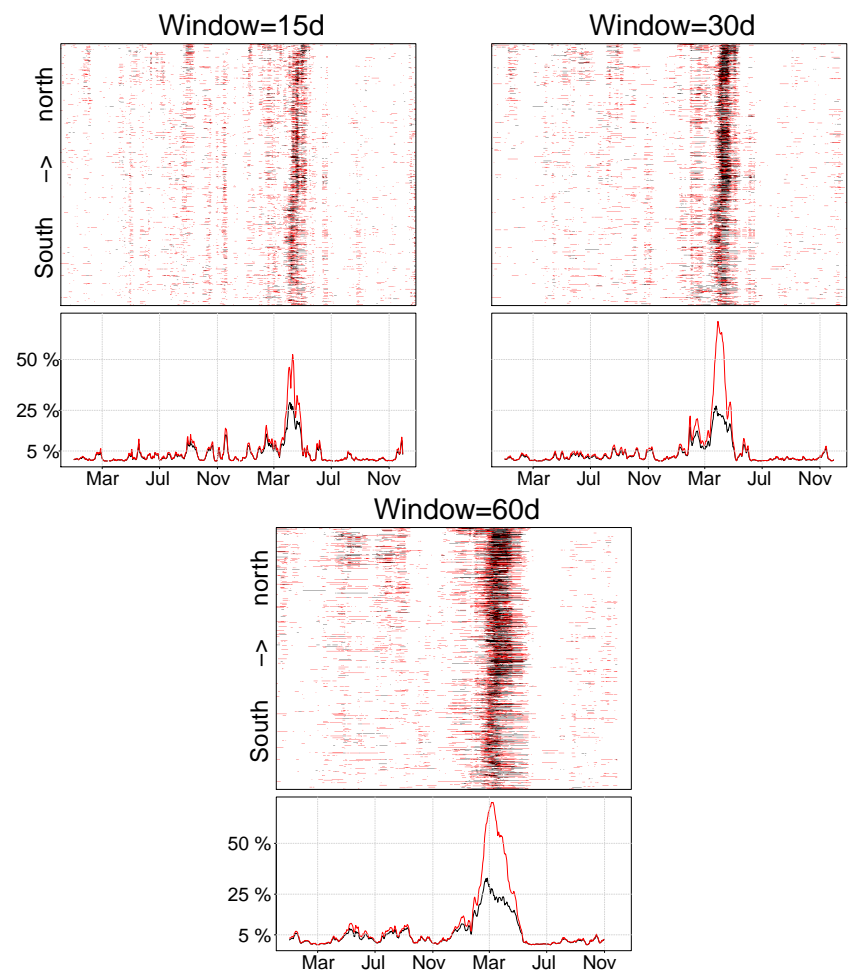

Figure 4. Latitudinal distribution (top panels) and total fraction (bottom panels) of stations with significant coincidence rates (red: $\alpha=0.05$; black: $\alpha=0.01$ ) between very early lilac flowering and extremely high window-mean temperatures for three different window sizes. The $x$ axes refer to the starting date of a window. The dashed horizontal lines at $5 \%$ in the lower panels highlight the employed group-significance criterion. be expected from the tolerable number of false positives in our testing procedure (Fig. 4).

This effect is mainly present at the more northern stations. We will further discuss possible explanations of these findings in Sect. 5.

Following upon the previous findings for lilac, Fig. 5 summarizes the corresponding results for the flowering of the other three species (red lines). For convenience, we only show the results for two window sizes and without further latitudinal resolution (the corresponding, more detailed results can be found in the Supplement). For elder the maximum fraction of stations with significant coincidence rates arises (due to the generally later flowering of elder) between March and May. Later windows also show a few stations with significant coincidence rates due to the previously discussed test design. A clear latitudinal gradient is absent in the significance profile (see the Supplement). As an exception, for the windows between January and March with a window size of 60 days, again mainly the more northern stations show significant coincidence rates, exhibiting 1-2 peaks in the corresponding temporal profile around the previous year's May and September. The latter peak is especially pronounced for the 15-day windows.

The results for hawthorn closely resemble those obtained for elder, including a clear maximum in the fraction of stations with significant coincidence rates in late spring and no clear influence of latitude. However, the corresponding signal during May and September of the preceding year is less pronounced or not even visible at all. Only for the 15-day windows, significant coincidence rates with September temperatures at the northern stations are clearly beyond the expected number of false positives. 

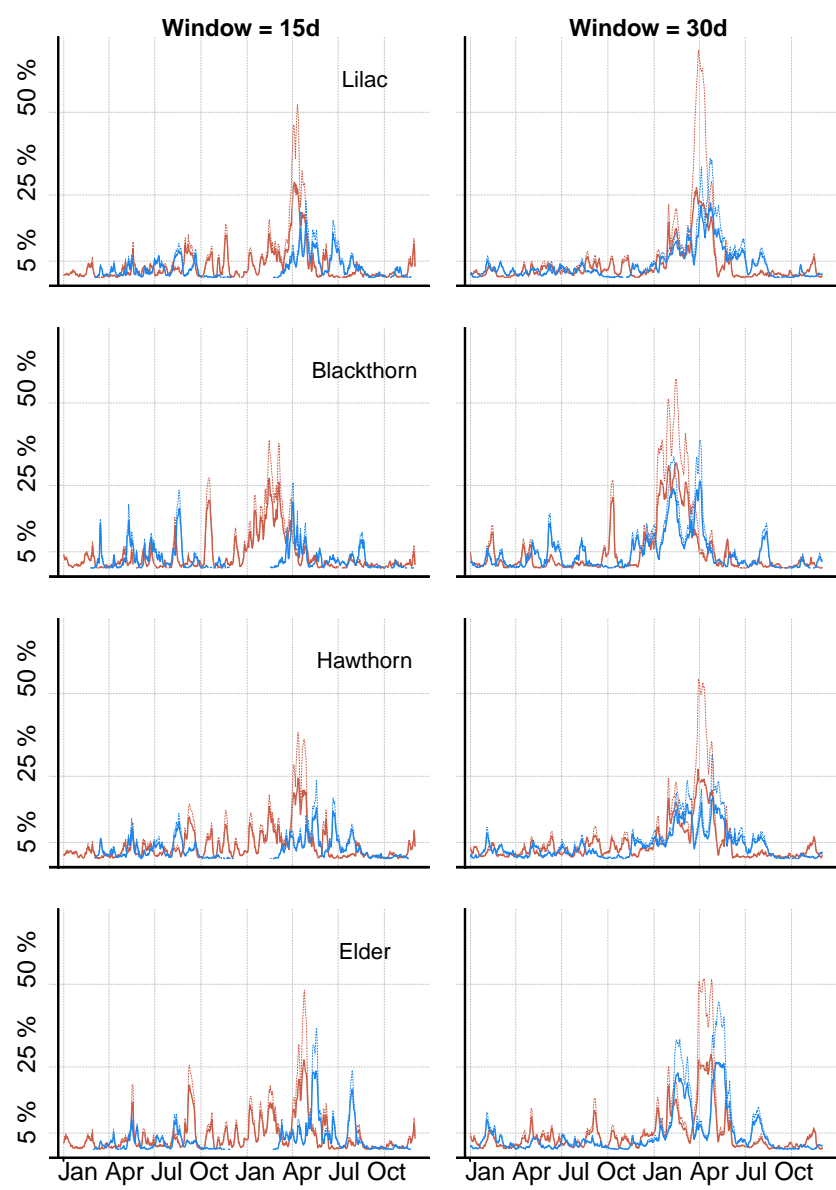

Figure 5. Fraction of stations with significant coincidence rates between extreme flowering dates and extreme window-mean temperature for the four shrub species and two different window sizes. The $x$ axes refer to the starting date of a window; the $y$ axes denote the percentage of stations that show significant coincidence rates for the specific window. Red (blue) lines refer to coincidences of extreme warm (cold) temperatures with extreme early (late) flowering at confidence levels of $\alpha=0.05$ (solid) and $\alpha=0.01$ (dotted), respectively.

Finally, the results for blackthorn are markedly shifted towards early spring, consistent with the generally earlier flowering of blackthorn in comparison to the three other shrub species. In contrast, the pertaining signal in the previous autumn is distinctively stronger in the 30-day window than for the other species.

\subsection{Coincidences with negative temperature extremes}

The blue lines in Fig. 5 display the results of event coincidence analysis between negative (cold) temperature extremes and late flowering. The general shape and intensity of the temporal profile of the number of stations with significant coincidence rates are similar to the results reported above for extremely positive seasonal temperature anomalies, yet slightly shifted towards later time windows. Our results do not show any significant peaks in the number of stations with statistically significant coincidence rates in the previous year for lilac, hawthorn and elder, while for blackthorn, even more distinct peaks in the previous year can be seen than for positive temperature extremes (at least for small windows). Likewise, the tendency of coincidences with temperature extremes in the previous year to be more pronounced at more northern latitudes (as observed for warm extremes) is not visible at all within the results for cold temperatures (see the Supplement). In turn, there is even an opposite tendency: for blackthorn, peaks in the previous year almost completely result from stations south of $50^{\circ} \mathrm{N}$. It is notable that this latitudinal distribution has the opposite direction in comparison with that observed for the latitudinal distribution of significant coincidence rates between very warm autumns and very early flowering dates.

\subsection{Coincidences with precipitation extremes}

As described in the Introduction, the impact of heavy or low rainfall amounts on flowering dates is not yet a fully understood topic. To contribute to this ongoing field of research, we have performed event coincidence analysis between extremely high/low precipitation amounts and extremely early/late flowering. For all four shrub species and all four possible extreme event combinations, we hardly ever find more than $5 \%$ of the stations showing significant coincidence rates (see the Supplement). Only two small exceptions are observed for blackthorn, but these are probably a result of the fact that very warm spring conditions normally originate from intense westerly circulation patterns, which are characterized by relatively high precipitation amounts in central Europe. For an explicit study of the latter relationship, multivariate extensions of event coincidence analysis would be required, the development of which is a subject of ongoing studies (Siegmund et al., 2016a). To this end, we conclude that there is no significant indication of a marked impact of precipitation extremes on the flowering of the four considered shrub species in Germany. Note that the productivity of German terrestrial ecosystems is commonly not limited by water availability. Hence, this result does not necessarily imply a similar absence of relationships for other species and/or regions, especially in situations where water stress can be a problem. We plan to address this question further in our future work.

\subsection{Combined effects of temperature and precipitation extremes}

Figure 6 illustrates the distribution of flowering dates for years that exhibit different combinations of early spring temperature and precipitation extremes (with early spring being defined here as the same time windows as before). Specifically, we consider "warm" ("cold") as a situation with the mean spring temperature (as in Fig. 3) being higher (lower) 

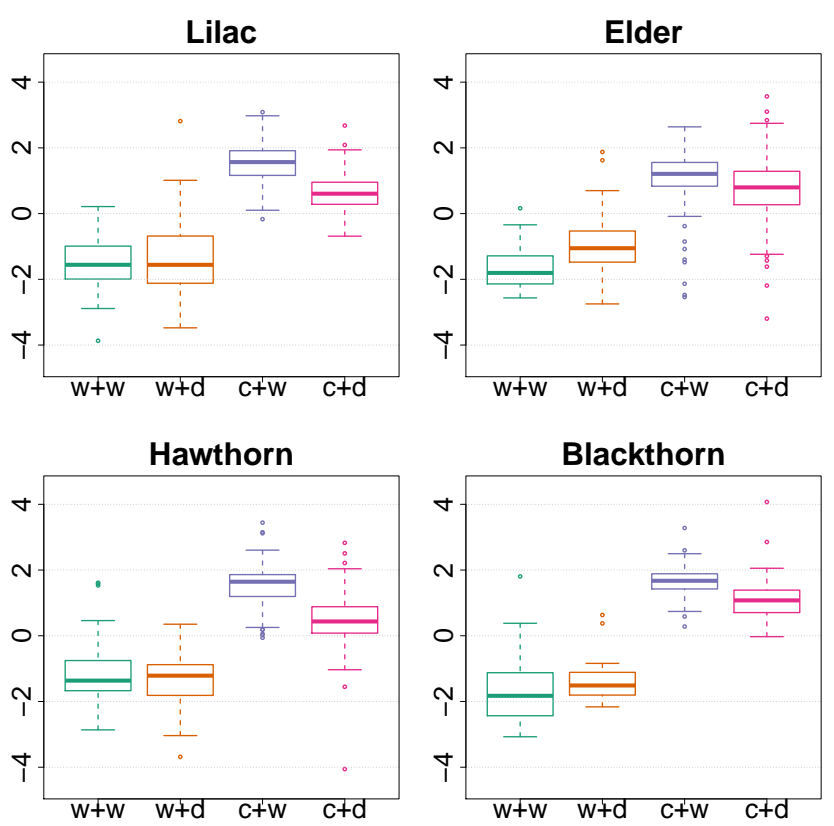

Figure 6. Distribution of flowering dates (standard box plots) for years that exhibit four different combinations of extreme meteorological conditions during early spring $(\mathrm{w}+\mathrm{w}$ : warm and wet; $\mathrm{w}+\mathrm{d}$ : warm and dry; $\mathrm{c}+\mathrm{w}$ : cold and wet; $\mathrm{c}+\mathrm{d}$ : cold and dry).

than the 90th (10th) percentile. Similarly, "wet” (“dry”) conditions are defined as years with spring precipitation sums higher (lower) than the respective percentile.

The results obtained in this way are very similar for all four shrub species. Warm and wet as well as warm and dry spring conditions generally lead to similarly early flowering dates (with exception of elder, where warm and wet conditions have a clearly stronger impact than warm and dry years). Following cold and wet spring conditions, the flowering dates of all four species are heavily delayed, with the effect being somewhat stronger than for very cold and dry conditions. Taken together, this analysis again confirms the minor-to-negligible influence of extremes in water availability on flowering dates in the study region. It should be noted, however, that in each individual combination of temperature and precipitation extremes, we also find cases where the flowering dates show a deviation from normal that is the opposite of what would be expected. The latter is particularly evident for elder and indicates that early spring mean temperature and precipitation sum alone cannot fully describe the plant's flowering dates, but it calls for the consideration of further covariates (like temperature/precipitation in different time windows as well as other meteorological factors not available in the studied data set). The latter observation has a potential relevance for developing improved statistical models for anticipating flowering dates but possibly also other phenological phases. A further detailed investigation of this problem is, however, beyond the scope of the present work.

\section{Discussion}

The results displayed in Figs. 4 and 5 suggest that event coincidence analysis is (in combination with a sliding window approach) indeed an appropriate technique to identify periods during or prior to the growing season, where extreme temperatures or precipitation sums are statistically related to extreme flowering dates. To our best knowledge, no similar analysis has been performed so far. In turn, all previous studies on possible relations between climate variables and flowering times have been based on linear correlation (Ahas et al., 2000; Sparks et al., 2000; Menzel, 2003). While correlations take all parts of the distributions of the two considered observables into account, event coincidence analysis exclusively focuses on the extremes, ignoring all other values. Although it is widely known that early spring temperatures strongly influence flowering dates, the specific validity of such a relationship for extreme values cannot be concluded from classical correlation analysis (see the Supplement for a detailed discussion). In turn, our methodological approach showed that the relationship does indeed also apply to the extreme values of temperature and flowering time for a significant subset of the investigated stations.

Another notable observation of this study is that positive temperature extremes (warm periods) that coincide with early flowering do not occur arbitrarily early in the year. This general finding is valid for all four analysed shrub species. However, an important exception can be seen at some stations in the very north of the study region and thus close to the North and Baltic seas. For these stations, the time windows for which significant coincidence rates between temperature and flowering date are evident, reach much further into late winter. This observation could result from the regulating effect of these two large water bodies, the large heat capacity of which allows maintaining relatively warm but not necessarily extreme air temperatures (especially during night-time, i.e. suppressing freezing conditions during winter time) for a considerable period of time. As a consequence, an extremely warm period in, for example, January can have a persistent effect on terrestrial ecosystems in coastal regions over the following weeks, resulting in coincidences between positive January window-mean temperature extremes and early flowering. This effect also explains why the prolonged significance peaks (late winter until late spring) of the northernmost stations in Fig. 4 are mainly visible for the longer time windows, since only long-lasting unusually warm conditions are stored for a substantial amount of time. A similar time-lagged regulatory effect of large water bodies on air temperatures (mediated via the long-term memory of sea-surface temperatures) is well known for El Niño events (Kumar and Hoerling, 2003). It was also found that North Atlantic temperature anomalies can influence atmospheric conditions in the following seasons with time lags of up to several months (Wedgbrow et al., 2002; Iwi et al., 2006). 
Our analysis also reveals another important observation: for lilac, elder, hawthorn and blackthorn (Fig. 4), we find a small but noticeable signature of coincidences between very warm 15-day windows during early September and very early flowering in the following year. This feature is relatively weakly expressed in comparison to the effect of spring temperature anomalies directly preceding the flowering but still far larger than the expected tolerable false positive rate of our test setting. Indications of the existence of such significant statistical relationships between flowering and temperatures of the previous growing season have already been found by, e.g., Sparks et al. (2000) for autumn crocus and by Fitter et al. (1995), Luterbacher et al. (2007) and Crimmins et al. (2010) for various other plant species. Heide (2003) reported that autumn temperatures were significantly correlated with the number of days to bud burst in the subsequent spring in field experiments with a range of latitudinal birch populations at $60^{\circ} \mathrm{N}$. However, the direction of the influence of warm autumn temperatures on the timing of flowering seems to strongly depend on plant species and geographical conditions like elevation (Crimmins et al., 2010). Cook et al. (2012) reported divergent responses of different plant species to spring and winter warming: "(i) apparent nonresponders are indeed responding to warming, but their responses to fall/winter and spring warming are opposite in sign and of similar magnitude; (ii) observed trends in first flowering date depend strongly on the magnitude of a given species' response to fall/winter vs. spring warming; and (iii) inclusion of fall/winter temperature cues strongly improves hindcast model predictions of long-term flowering trends compared with models with spring warming only". In the context of our present study, this accumulated evidence raises confidence that the findings reported here are not just statistical artifacts resulting from the auto-correlation of temperature time series but plant physiologically meaningful. In order to further address this question, future studies should explicitly address the potential influence of auto-correlations in more detail, calling for a methodological extension of event coincidence analysis conditioning on previous events (in a similar spirit as partial correlations or conditional mutual information; see, e.g., Balasis et al., 2013; Siegmund et al., 2016a).

We have also considered the combined effects of very cold/warm and wet/dry conditions. Notably, the delaying impact of very cold conditions appears more effective in combination with very wet than with very dry conditions. This finding was not to be expected from independent analyses of temperature and precipitation influences as commonly considered in the literature (Zscheischler et al., 2014), where in our case precipitation was found not to have any statistically significant effect. However, it may act as a potentially relevant modulator of the effect of extreme temperature on plant phenology. In the specific case of shrub flowering studied in this work, we hypothesize that the additionally delaying effect of wet conditions could be related to snow cover. In fact, prolonged snow cover has widely been found to delay plant

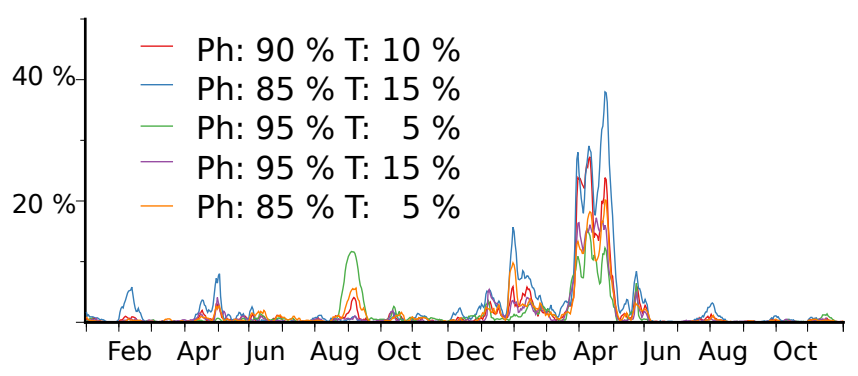

Figure 7. Fraction of stations with significant coincidence rates $(\alpha=0.05)$ among all phenological stations for 30-day windows and five different threshold combinations of extremely warm windowmean temperature and extremely early elder flowering. Note that the red line is the same as the bold red line displayed in Fig. 5, lower right panel.

development including flowering dates for several species in the Arctic (Cooper et al., 2011; Semenchuk et al., 2013) and alpine regions (Inouye, 2008; Dunne et al., 2003).

In contrast, for very warm spring conditions, very high or very low precipitation sums have little additional effect for lilac, hawthorn and blackthorn, while the effect of warm conditions appears to be slightly reduced during years with dry springs for elder. In all cases, it has to be noted that for all four possible combinations of temperature and precipitation extremes, the distribution of flowering dates of the respective years has been very broad and includes in most cases also situations that appear to contradict the previously reported mean effect. Further work should clarify to what extent this observation can be related to site-specific effects.

A potential drawback of event coincidence analysis applied to non-binary data could be a dependence of the results on the threshold used for the definition of an extreme. In this study, we used the 90th and 10th percentiles for temperature, precipitation and flowering time. In order to further demonstrate the qualitative robustness of our results, Fig. 7 recalls the results of Fig. 5 (right panel, second row) with five different threshold definitions. The obtained results show that although the absolute number of stations with significant coincidence rates varies among the different threshold combinations (as expected from the definitions of events and coincidences), the general temporal profile qualitatively remains the same for most windows. In most cases the observed numbers of stations with significant coincidence rates are larger for less restrictive thresholds. As a notable exception, regarding the relevance of warm autumn temperature in the previous year, we find an opposite behaviour, i.e. the event coincidence analysis using a more restrictive threshold (green line in Fig. 7) results in a higher number of stations with significant coincidence rates than the same analysis employing more conservative thresholds (e.g. red line in Fig. 7), indicating that this observation could have been caused by only a few events per station within the considered window of observations. Hence, whereas the relationship between ex- 
tremely positive temperature anomalies in spring and early flowering appears to consistently apply for different event magnitudes, for the previous autumn, the strongest positive anomalies could have an over-proportional relevance for the emergence of very early elder flowering.

\section{Conclusions}

In summary, the first-time application of the modern statistical concept of event coincidence analysis to phenological data revealed a clear statistical relationship between extremely warm spring temperatures and very early flowering dates of lilac, elder, hawthorn and blackthorn, as well as between extremely cold temperatures in spring and extremely late flowering dates. Although this relationship is not evident for all investigated study sites individually, the observed coincidences are quite homogeneously distributed over the study area (see the Supplement).

In addition to the expected relevance of spring temperatures, we identified a period during the previous year's autumn, where extremely warm temperatures significantly coincide with an extremely early flowering in the subsequent year. Although the signatures of this period are not very strong, they are clearly visible. Our study revealed that this effect becomes even stronger when more restrictive threshold definitions are used. In contrast to the confirmed dependence of early and late flowering events on temperature extremes, our analysis did not identify similar marked statistical relationships between extreme precipitation amounts and the timing of flowering unless the precipitation anomalies (in both directions) co-occur with very cold temperatures.

To answer the research questions formulated in the Introduction, we conclude that extremely high (low) temperatures do significantly coincide with extremely early (late) flowering, especially if the extreme period appears during early spring. All four analysed shrub species show the same qualitative behaviour and only differ in the timing, according to their typical flowering time. The specific findings differ somewhat by region, but an easily explainable pattern or spatial clustering of stations with significant coincidence rates could not be found. Our results further support the outcomes of previous studies by underlining the fact that known interdependencies between meteorological variables and flowering dates do not only cover the bulk of their corresponding empirical distributions (as highlighted by studies using linear correlation analysis) but for the tails (i.e. extreme conditions).

To this end, our work presented here has served as a pilot study demonstrating the application of event coincidence analysis in the context of plant phenology. A systematic extension of the obtained results to more species, more phenological phases and more meteorological covariates appears reasonable. In order to cope with data from study sites where even fewer years of observations are available, recently de- veloped group-significance tests for event coincidence analysis (Donges et al., 2016) are ready for application. In a similar spirit, multivariate extensions of this approach (Siegmund et al., 2016b) allow the systematic study of combined impacts of (simultaneous or mutually time-shifted) extremes in different meteorological variables. For the latter purpose (especially the consideration of effects of water availability), the meteorological data set used in this work needs to be systematically extended.

We emphasize that besides providing directly usable information for forest and agricultural management purposes, future extended studies along the lines of the present work do also have great potential value for biogeographical model development. To date, the usability of information about extreme weather impacts on flowering dates for numerical ecosystem models like the Lund-Potsdam-Jena Dynamic Global Vegetation Model (LPJ; Sitch et al., 2003) is strongly limited by the fact that in most cases, these models use growing degree days and corresponding temperature sums for the calculation of phenological phases. Specific extreme events of limited temporal extent like those investigated in our study can commonly not be considered. Furthermore, the parameterizations of shrubs normally does not distinguish between different species. Here, further systematic empirical analyses may provide valuable input to refining these parameterizations.

The findings of this study underline the risk of potential phenological mismatches due to temperature extremes, at least from the plant-ecological perspective. In future studies, it will be especially important to further investigate possible delayed influences of extremely warm temperatures on flowering dates of the following growing season.

\section{Data availability}

The phenology data used in this study are provided by the German weather service DWD and are available at http: //www.dwd.de/phaenologie. For meteorological information we used temperature and precipitation time series produced and provided by Österle et al. (2006), which are available upon request to peterh@pik-potsdam.de.

\section{The Supplement related to this article is available online at doi:10.5194/bg-13-5541-2016-supplement.}

Acknowledgements. This study was conducted within the framework of the BMBF Young Investigators Group "CoSy-CC" Complex Systems Approaches to Understanding Causes and Consequences of Past, Present and Future Climate Change" (grant no. 01LN1306A) funded by the German Federal Ministry for Education and Research (BMBF). J. F. Siegmund additionally acknowledges the Evangelisches Studienwerk Villigst for providing 
financial support. J. F. Donges has been funded by the Stordalen Foundation (via the Planetary Boundary Research Network PB.net), the Earth League's EarthDoc program and the BMBF via the project GLUES. Stimulating discussions with Diego Rybski are gratefully acknowledged. Event coincidence analysis was performed using the R package CoinCalc (Siegmund et al., 2016b) available at https://github.com/JonatanSiegmund/CoinCalc.git.

Edited by: M. Weintraub

Reviewed by: two anonymous referees

\section{References}

Ahas, R., Jaagus, J., and Aasa, A.: The phenological calendar of Estonia and its correlation with mean air temperature, Int. J. Biometeorol., 44, 159-166, 2000.

Atkinson, M. and Atkinson, E.: Sambucus nigra L., J. Ecol., 90, 895-923, 2002.

Augspurger, C.: Spring 2007 warmth and frost: phenology, damage and refoliation in a temperate deciduous forest, Funct. Ecol., 23, 1031-1039, 2009.

Balasis, G., Donner, R., Potirakis, S., Runge, J., Papadimitriou, C., Daglis, I., Eftaxias, K., and Kurths, J.: Statistical Mechanics and Information-Theoretic Perspectives on Complexity in the Earth System, Entropy, 15, 4844-4888, 2013.

Barriopedro, D., Fischer, E., Luterbacher, J., Trigo, R., and GarciaHerrera, R.: The Hot Summer of 2010: Redrawing the Temperature Record Map of Europe, Science, 332, 220-224, 2011.

Burkle, L., Marlin, J., and Knight, T.: Plant-Pollinator Interactions over 120 Years: Loss of Species, Co-Occurrence, and Function, Science, 339, 1611-1615, 2013.

Cleland, E., Chuine, I., Menzel, A., Mooney, H., and Schwartz, M.: Shifting plant phenology in response to global change, Trends Ecol. Evol., 22, 357-365, 2007.

Cook, B., Wolkovich, E., and Parmesan, C.: Divergent responses to spring and winter warming drive community level flowering trends, P. Natl. Acad. Sci. USA, 109, 9000-9005, 2012.

Cooper, E., Dullinger, S., and Semenchuk, P.: Late snowmelt delays plant development and results in lower reproductive success in the High Arctic, Plant Sci., 180, 157-167, 2011.

Coumou, D. and Rahmstorf, S.: A decade of weather extremes, Nature Climate Change, 2, 491-496, 2012.

Crimmins, T., Crimmins, M., and Bertelsen, C.: Complex responses to climate drivers in onset of spring flowering across a semi-arid elevation gradient, J. Ecol., 98, 1042-1051, 2010.

Deutscher Wetterdienst, Offenbach: DWD Klimastationen: Daten der Klimastationen des Deutschen Wetterdienstes, 2009.

Donges, J., Donner, R., Trauth, M., Marwan, N., Schellnhuber, H.J., and Kurths, J.: Nonlinear detection of paleoclimate-variability transitions possibly related to human evolution, P. Natl. Acad. Sci. USA of the USA, 108, 20422-20427, 2011.

Donges, J., Schleussner, C.-F., Siegmund, J., and Donner, R.: Event coincidence analysis for quantifying statistical interrelationships between event time series: on the role of flood events as triggers of epidemic outbreaks, Eur. Phys. J.-Spec. Top., 225, 471-487, 2016.
Duffey, E., Morris, M., Sheail, J., Ward, L., Wells, D., and Wells, T.: Grassland Ecology and Wildlife Management, Chapman and Hall, 1974.

Dunne, J., Harte, J., and Taylor, K.: Subalpine meadow flowering phenology responses to climate change: Integrating experimental and gradient methods, Ecol. Monogr., 73, 69-86, 2003.

Easterling, D., Meehl, G., Parmesan, C., Changnon, S., Karl, T., and Mearns, L.: Climate extremes: observations, modeling, and impact, Science, 289, 2069-2074, 2000.

Fischer, E., Seneviratne, S., Luethi, D., and Schaer, C.: Contribution of land-atmosphere coupling to recent European summer heat waves, Geophys. Res. Lett., 34, L0607, doi:10.1029/2006GL029068, 2007.

Fitter, A., Fitter, R., Harris, I., and Williamson, M.: Relationships between first flowering date and temperature in the flora of a locality in central England, Funct. Ecol., 9, 55-60, 1995.

Frank, D., Reichstein, M., Bahn, M., Thonicke, K., Frank, D., Mahecha, M., Smith, P., Van der Velde, M., Vicca, S., Babst, F., Beer, C., Buchmann, N., Canadell, J., Ciais, P., Cramer, W., Ibrom, A., Miglietta, F., Poulter, B., Rammig, A., Seneviratne, S., Walz, A., Wattenbach, M., Zavala, M., and Zscheischler, J.: Effects of climate extremes on the terrestrial carbon cycle: concepts, processes and potential future impacts, Glob. Change Biol., 21, 7861-2880, 2015.

Garcia-Herrera, R., Diaz, J., Trigo, R., Luterbacher, J., and Fischer, E.: A Review of the European Summer Heat Wave of 2003, Critical Reviews in Environmental Science and Technology, 40, 267 306, 2010.

Gudmundsson, L. and Seneviratne, S. I.: European drought trends, Proc. IAHS, 369, 75-79, doi:10.5194/piahs-369-75-2015, 2015.

Haylock, M. and Goodess, C.: Interannual variability of european extreme winter rainfall and links with mean large-scale circulation, Int. J. Climatol., 24, 759-776, 2004.

Heide, O.: High autumn temperature delays spring bud burst in boreal trees, counterbalancing the effect of climatic warming, Tree Physiol., 23, 931-936, 2003.

Inouye, D.: Effects of climate change on phenology, frost damage, and floral abundance of montane wildflowers, Ecology, 89, 353362, 2008.

IPCC: Climate Change 2013: The Physical Science Basis. Contribution of Working Group I to the Fifth Assessment Report of the Intergovernmental Panel on Climate Change, Cambridge University Press, Cambridge, United Kingdom and New York, NY, USA, 2013.

Iwi, A., Sutton, R., and Norton, W.: Influence of May Atlantic Ocean initial conditions on the subsequent North Atlantic winter climate, Q. J. Roy. Meteorol. Soc., 132, 2977-2999, 2006.

Jacobs, J., Clak, S., Denholm, I., Goulson, D., Stoate, C., and Osborne, J.: Pollination biology of fruit-bearing hedgerow plants and the role of flower-visiting insects in fruit-set, Ann. Bot., 104, 1397-1404, 2009.

Jentsch, A., Kreyling, J., and Beierkuhnlein, C.: A new generation of climate change experiments: events not trends, Front. Ecol. Environ., 5, 365-374, 2007.

Jentsch, A., Kreyling, J., Boettcher-Treschkow, J., and Beierkuhnlein, C.: Beyond gradual warming: extreme weather events alter flower phenology of European grassland and heath species, Glob. Change Biol., 15, 837-849, 2009. 
Kudo, G. and Ida, T.: Early onset of spring increases the phenological mismatch between plants and pollinators, Ecology, 94, 2311-2320, 2013.

Kumar, A. and Hoerling, M.: The nature and causes for the delayed atmospheric response to El Nino, J. Climate, 16, 1391-1403, 2003.

Kundzewicz, Z., Radziejewski, M., and Pinskwar, I.: Precipitation extremes in the changing climate of Europe, Clim. Res., 31, 5158, 2006.

Kysely, J., Gaal, L., Beranova, R., and Plavcova, E.: Climate change scenarios of precipitation extremes in Central Europe from ENSEMBLES regional climate models, Theor. Appl. Climatol., 104, 529-542, 2011.

Law, B., Mackowski, C., Schoer, L., and Tweedie, T.: Flowering phenology of myrtaceous trees and their relation to climatic, environmental and disturbance variables in northern New South Wales, Aust. Ecol., 25, 160-178, 2000.

Lupikasza, E., Hansel, S., and Matschullat, J.: Regional and seasonal variability of extreme precipitation trends in southern Poland and central-eastern Germany 1951-2006, Int. J. Climatol., 31, 2249-2271, 2011.

Luterbacher, J., Dietrich, D., Xoplaki, E., Grosjean, M., and Wanner, H.: European seasonal and annual temperature variability, trends, and extremes since 1500, Science, 303, 1409-1503, 2004.

Luterbacher, J., Lininger, M., Menzel, A., Estrella, N., Della-Marta, P., Pfister, C., Rutishauser, T., and Xoplaki, E.: Exceptional European warmth of autumn 2006 and winter 2007: Historical context, the underlying dynamics, and its phenological impacts, Geophys. Res. Lett., 34, L12704, doi:10.1029/2007GL029951, 2007.

McKinney, A., CaraDonna, P., Inouye, D., Barr, B., Bertelsen, C., and Waser, N.: Asynchronous changes in phenology of migrating Broad-tailed Hummingbirds and their early-season nectar resources, Ecology, 93, 1987-1993, 2012.

Menzel, A.: Plant phenological anomalies in Germany and their relation to air temperature and NAO, Climatic Change, 57, 243263, 2003.

Menzel, A., Seifert, H., and Estrella, N.: Effects of recent warm and cold spells on European plant phenology, Int. J. Biometeorol., 55, 921-932, 2011.

Nagy, L., Kreyling, J., Gellesch, E., Beierkuhnlein, C., and Jentsch, A.: Recurring weather extremes alter the flowering phenology of two common temperate shrubs, Int. J. Biometeorol., 57, 579588, 2013.

Österle, H., Werner, P., and Gerstengarbe, F.: Qualitätsprüfung, Ergänzung und Homogenisierung der täglichen Datenreihen in Deutschland, 1951-2003: ein neuer Datensatz, in: 7. Deutsche Klimatagung, Klimatrends: Vergangenheit und Zukunft, 9-11 Oktober 2006, 2006.

Parmesan, C.: Ecological and evolutionary responses to recent climate change, Annual Review of Ecology, Evolution and Systematics, 37, 637-669, 2006.

Parmesan, C., Root, T., and Willig, M.: Impacts of Extreme Weather and Climate on Terrestrial Biota, B. Am. Meteorol. Soc., 81, 444-449, 2000.

Perneger, T.: What's wrong with Bonferroni adjustments, British Medical Journal, 316, 1236-1238, 1998.

Petoukhov, V., Rahmstorf, S., Petri, S., and Schellnhuber, H.J.: Quasiresonant amplification of planetary waves and recent
Northern Hemisphere weather extremes, P. Natl. Acad. Sci. USA of the USA, 110, 5336-5341, 2013.

Post, E. and Stenseth, N.: Climate Variability, Plant Phenology and Northern Ungulates, Ecology, 80, 1322-1339, 1999.

Prieto, P., Penuelas, J., Ogaya, R., and Estiarte, M.: Precipitationdependent flowering of Globularia alypum and Erica multiflora in Mediterranean shrubland under experimental drought and warming, and its inter-annual variability, Ann. Bot., 102, 275285, 2008.

Rafferty, N., CaraDonna, P., and Bronstein, J.: Phenological shifts and the fate of mutualisms, Oikos, 124, 14-21, 2015.

Rahmstorf, S. and Coumou, D.: Increase of extreme events in a warming world, P. Natl. Acad. Sci. USA, 108, 17905-17909, 2011.

Rajczak, J. P. P. and Schar, C.: Projections of extreme precipitation events in regional climate simulations for Europe and the Alpine Region, J. Geophys. Res.-Atmos., 118, 3610-3626, 2013.

Rammig, A., Wiedermann, M., Donges, J. F., Babst, F., von Bloh, W., Frank, D., Thonicke, K., and Mahecha, M. D.: Coincidences of climate extremes and anomalous vegetation responses: comparing tree ring patterns to simulated productivity, Biogeosciences, 12, 373-385, doi:10.5194/bg-12-373-2015, 2015.

Reichstein, M., Bahn, M., Ciais, P., Frank, D., Mahecha, M., Seneviratne, S., Zscheischler, J., Beer, C., Buchmann, N., Frank, D., Papale, D., Rammig, A., Smith, P., Thonicke, K., van der Velde, M., Vicca, S., Walz, A., and Wattenbach, M.: Climate extremes and the carbon cycle, Nature, 500, 287-295, 2013.

Reyer, C., Leuzinger, S., Rammig, A., Wolf, A., Bartholomeus, R., Bonfante, A., Lorenz, F., Dury, M., Glonning, P., Jaoude, R., Klein, T., Kuster, T., Martinis, M., Niedrist, G., Riccardi, M., Wohlfahrt, G., Angelis, P., DeDato, G., Francous, L., Menzel, A., and Pereira, M.: A plant's perspective of extremes: terrestrial plant responses to changing climatic variability, Glob. Change Biol., 19, 75-89, 2013.

Rutishauser, T., Luterbacher, J., Defila, C., Frank, D., and Wanner, H.: Swiss spring plant phenology 2007: Extremes, a multicentury perspective, and changes in temperature sensitivity, Geophys. Res. Lett., 35, L05703, doi:10.1029/2007GL032545, 2008.

Rybski, D., Holstern, A., and Kropp, J.: Towards a unified characterization of phenological phases: Fluctuations and correlations with temperature, Physica A, 390, 680-688, 2011.

Schaer, C., Vidale, P., Luethi, D., Frei, C., Haeberli, C., Liniger, M., and Appenzeller, C.: The role of increasing temperature variability in European summer heatwaves, Nature, 427, 332-336, 2004.

Schleip, C., Ankerst, D., Bock, A., Estrella, N., and Menzel, A.: Comprehensive methodological analysis of long-term changes in phenological extremes in Germany, Glob. Change Biol., 18, 2349-2364, 2012.

Semenchuk, P., Elberling, B., and Cooper, E.: Snow cover and extreme winter warming events control flower abundance of some, but not all species in high arctic Svalbard, Ecol. Evol., 3, 25862599, 2013.

Seneviratne, S. I., Nicholls, N., Easterling, D., Goodess, C. M., Kanae, S., Kossin, J., Luo, Y., Marengo, J., McInnes, K., Rahimi, M., Reichstein, M., Sorteberg, A., Vera, C., and Zhang, X.: Changes in climate extremes and their impacts on the natural physical environment, in: Managing the Risks of Extreme Events and Disasters to Advance Climate Change Adaptation edited by: Field, C. B., Barros, V., Stocker, T. F., Qin, D., Dokken, D. J., 
Ebi, K. L., Mastrandrea, M. D., Mach, K. J., Plattner, G.-K., Allen, S. K., Tignor, M., and Midgley, P. M., A Special Report of Working Groups I and II of the Intergovernmental Panel on Climate Change (IPCC), Cambridge University Press, Cambridge, UK, and New York, NY, USA, 109-230, 2012.

Shaffer, J.: Multiple Hypothesis Testing, Annu. Rev. Psychol., 46, 561-584, 1995.

Siegmund, J., Sanders, T., Heinrich, I., van der Maaten, E., Simard, S., Helle, G., and Donner, R.: Meteorological Drivers of Extremes in Daily Stem Radius Variations of Beech, Oak, and Pine in Northeastern Germany: An Event Coincidence Analysis, Front. Plant Sci., 7, 733, 2016a.

Siegmund, J., Siegmund, N., and Donner, R.: CoinCalc - A new $\mathrm{R}$ package for quantifying simultaneities of event (time) series, arXiv:1603.05038, 2016b.

Sitch, S., Smith, B., Prentice, I., Arneth, A., Bondeau, A., Cramer, W., Kaplan, J., Levis, S., Lucht, W., Sykes, M., Thonicke, K., and Venevsky, S.: Evaluation of ecosystem dynamics, plant geography and terrestrial carbon cycling in the LPJ dynamic global vegetation model, Glob. Change Biol., 9, 161-185, 2003.

Southwood, T.: The Number of Insect Species Associated with Various Trees, J. Animal Ecol., 30, 1-8, 1961.

Sparks, T. and Menzel, A.: Observed changes in seasons: an overview, Int. J. Climatol., 22, 1715-1725, 2002.

Sparks, T., Jeffree, E., and Jeffree, C.: An examination on the relationship between flowering times and temperature at the national scale using long-term phenological records from the UK, Int. J. Biometeorol., 44, 82-87, 2000.

Spinoni, J., Naumann, G., and Vogt, J.: Spatial patterns of European droughts under a moderate emission scenario, Adv. Sci. Res., 12, 179-186, 2015a.
Spinoni, J., Naumann, G., Vogt, J., and Barbosa, P.: European drought climatologies and trends based on a multi-indicator approach, Global Planet. Change, 127, 50-57, 2015b.

Stott, P., Stone, D., and Allen, R.: Human contribution to the European heatwave of 2003, Nature, 432, 610-614, 2004.

Tank, K. and Konnen, G.: Trends in indices of daily temperature and precipitation extremes in Europe, 1946-99, J. Climate, 16, 3665-3680, 2003.

Trenberth, K. and Fasullo, J.: Climate extremes and climate change: The Russian heat wave and other climate extremes of 2010, J. Geophys. Res.-Atmos., 117, D17103, doi:10.1029/2012JD018020, 2012.

Wedgbrow, C., Wilby, R. L., Fox, H., and O'Hare, G.: Prospects for seasonal forecasting of summer drought and low river flow anomalies in England and Wales, Int. J. Climatol., 22, 219-236, 2002.

WMO: Standardized Precipitation Index User Guide, Geneva, 2012.

Zimmermann, N., Yoccoz, N., Edwards, T., Meier, E., Thuiller, W., Guisan, A., Schmatz, D., and Pearman, P.: Climatic extremes improve predictions of spatial patterns of tree species, P. Natl. Acad. Sci. USA, 17, 19723-19728, 2009.

Zscheischler, J., Michalak, A., Schwalm, C., Mahecha, M., Huntzinger, D., Reichstein, M., Berthier, G., Ciais, P., Cook, R., El-Masri, B., Huang, M., Ito, A., Jain, A., King, A., Lei, H., Lu, C., Mao, J., Peng, S., Poulter, B., Ricciuto, D., Shi, X., Tao, B., Tian, H., Viovy, N., Wang, W., Wei, Y., Yang, J., and Zeng, N.: Impact of large-scale climate extremes on biospheric carbon fluxes: An intercomparison based on MsTMIP data, Global Biochem. Cy., 28, 585-600, 2014. 